\title{
XLI CONGRESO CHILENO DE ENFERMEDADES RESPIRATORIAS
}

5 al 8 de Noviembre del 2008

Hotel Termas de Chillán - Chillán

\section{COMITÉ ORGANIZADOR}

\begin{tabular}{|c|c|}
\hline Presidente & : Dr. Fernando Rivas B. \\
\hline Vice-Presidente & : Dr. Fernando Descalzi M. \\
\hline Past- President & : Dr. Raúl Corrales V. \\
\hline Secretario & : Dra. Patricia Schönffeldt G. \\
\hline Tesorero & : Dr. Edgardo Grob B. \\
\hline Coordinador Adulto & : Dra. Carolina Herrera C. \\
\hline Coordinadora Pediátrica & : Dra. Isabel Valdés I. \\
\hline Coordinador Cirugía & : Dr. Raimundo Santolaya C. \\
\hline Coordinadores Regionales & $\begin{array}{l}\text { : Dr. Hugo Hidalgo T. } \\
\text { Dr. Carlos Ortega R. }\end{array}$ \\
\hline Comité Científico & $\begin{array}{l}\text { Drs. Patricia Díaz A. (Coordinadora) } \\
\text { Gisella Borzone T. } \\
\text { Edgardo Cruz M. } \\
\text { Manuel Oyarzún G. } \\
\text { María Angélica Palomino M. } \\
\text { Gonzalo Valdivia C. } \\
\text { María Teresa Vicencio A. }\end{array}$ \\
\hline
\end{tabular}

Revisores Invitados : Drs. Viviana Aguirre C. Cecilia Álvarez G.

Fidel Avendaño C. Sergio Bello S.

Manuel Barros M. Gisella Borzone T.

María Lina Boza C. Edgardo Carrasco C.

Juan Céspedes G. José Miguel Clavero

Gustavo Contreras Raúl Corrales V.

Carolina Cruz B. Edgardo Cruz M.

Sara Chernilo S. Orlando Díaz P.

Patricia Díaz A. Victorino Farga C.

Ramiro González V. Sergio González B.

Mónica Gutiérrez C. Mónica Gutiérrez N.

Ana María Herrera Carolina Herrera C.

Patricio Jiménez $P$. Jorge Jorquera A.

Marcela Linares P. Pablo Marcone E.

Manuel Meneses C. Henry Olivi R.

Manuel Oyarzún G. María Angélica Palomino M.

María Angélica Pérez Julio Pertuzé R.

Ricardo Pinto M. Francisco Prado A.

Guillermo Ríos O. Juan Carlos Rodríguez D.

Fernando Saldías P. Patricia Schönffeldt G.

Ricardo Sepúlveda M. Carlos Ubilla P.

Alvaro Undurraga P. Gonzalo Valdivia C.

María Teresa Vicencio A. Fanor Villanueva Ch.

Mónica Zagolín B. 
INSTITUCIONES PATROCINANTES

Universidad de Valparaíso

Universidad de Santiago

Universidad de Los Andes

Universidad de La Frontera

Universidad de Concepción

Universidad de Chile

Universidad Austral de Chile

Sociedad Médica de Santiago

Sociedad Chilena de Pediatría

Sociedad Chilena de Alergia e Imnunología

Servicio de Salud Metropolitano Sur-Oriente

Servicio de Salud Metropolitano Sur

Servicio de Salud Metropolitano Oriente

Servicio de Salud Metropolitano Occidente

Servicio de Salud Metropolitano Norte

Servicio de Salud Metropolitano Central

Pontificia Universidad Católica de Chile

OMS/OPS en Chile

Ministerio de Salud

Ilustre Municipalidad de Santiago

Conacem

Universidad del Desarrollo

Servicio de Salud Concepción

Servicio de Salud Arauco

Servicio de Salud Talcahuano

Servicio de Salud Bío Bío

Hospital Clínico Regional - Guillermo Grant Benavente

Hospital Las Higueras

\section{INSTITUCIONES COLABORADORAS}

Air Liquide Chile S.A.

Astra Zeneca

Bayer S.A.

Bioscom Chile Ltda.

Boehringer Ingelheim

Covidien

GHC Chile

GlaxoSmithkline Chile

Grifols Chile S.A.

Indura S.A.

Laborario Luis Pasteur S.A.

Laboratorio Chile S.A.

Laboratorio Novartis Chile S.A.

Laboratorios Andrómaco S.A.

Laboratorios Recalcine S.A.

Laboratorios Saval S.A.

Merck Sharp \& Dohme

Pfizer Chile S.A.

Sanofi Aventis

Schering Plough Cia. Ltda.

Teveuci 


\section{INVITADOS INTERNACIONALES}

\section{Prof. Marlos De Souza Coelho MD}

Profesor Adjunto de Cirugía. Jefe de Servicio de Cirugía Torácica y Endoscopia Respiratoria, Pontificia Universidad Católica de Paraná, Curitiba, Brasil.

\section{Juan Carlos Figueroa-Casas MD}

Ex Presidente AAMR Rosario. Médico investigador (Estudio UPLIFT). Miembro de la Sociedad de Neumología Argentina, Rosario, Argentina.

\section{Kevin R. Flaherty MD, MS}

Associate Professor of Medicine. Associate Director Pulmonary/Critical Care Fellowship Program. Division of Pulmonary/Critical Care. University of Michigan Health System. Michigan, USA.

\section{Ronald Grossman MD.}

Professor University of Toronto, Respirologist, Credit Valley Hospital, University of Toronto. Mount Sinai Hospital, Ontario, Canadá.

\section{Héctor Gutiérrez MD.}

Associate Professor of Pediatrics, Division of Pediatric Pulmonary and Sleep Medicine, Director, UAB/ CHS Cystic Fibrosis Center, Director, Pediatric Pulmonology Fellowship Program, University of Alabama at Birmingham. Departament of Pediatric, Alabama, USA.

\section{Vicente Macian}

Enfermero supervisor del laboratorio de función pulmonar del Servicio de Neumología del Hospital U. La Fe de Valencia, miembro del Comité de Asuntos Profesionales, miembro del Comité Científico SEPAR, Valencia, España.

\section{Martyn R. Partridge MD.}

President of the British Thoracic Society. Professor of Respiratory Medicine in Imperial College London, Inglaterra.

\section{Moisés Rosenberg MD}

Director Hospital María Ferrer, Jefe Servicio Cirugía Torácica. Instituto Alexander Fleming, Buenos Aires, Argentina.

\section{Peter D. Sly MD.}

Head of Division Clinical Sciences. Director Clinical Research and Education, Princess Margaret Hospital for Children, Australia.

\section{Donald P. Tashkin MD.}

Professor of Medicine. David Geffen School of Medicine at UCLA, Los Angeles, USA. 


\title{
41 CONGRESO CHILENO DE ENFERMEDADES RESPIRATORIAS
}

5 al 8 de Noviembre del 2008

Hotel Termas de Chillán - Chillán

\author{
MIÉRCOLES 5 DE NOVIEMBRE \\ Coordinadores del día: \\ Coordinador Adulto: Dr. Cristián Ibarra \\ Coordinadora Pediatra: Dra. Rebeca Paiva
}

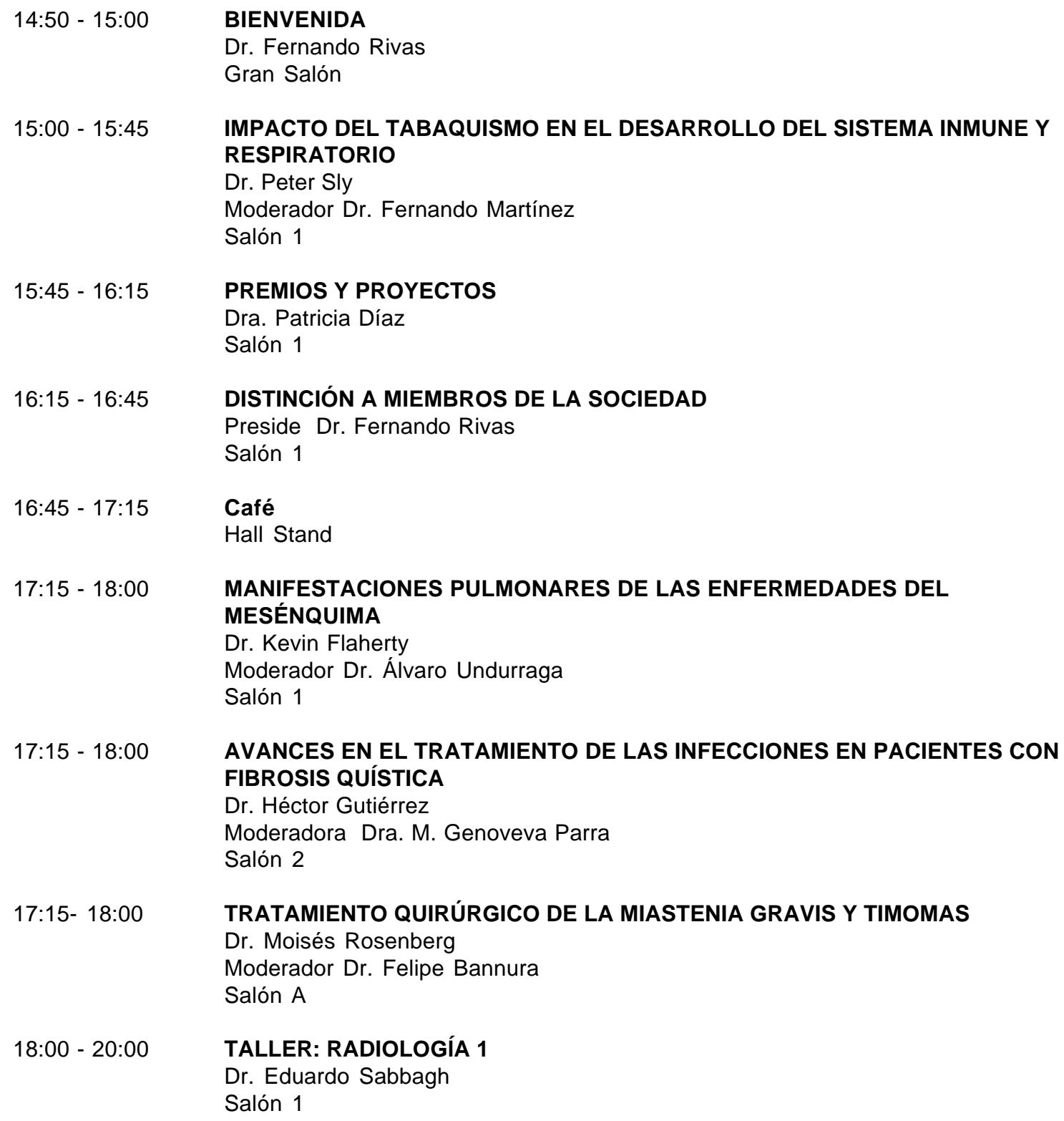


18:00 - 20:00

18:00 - 20:00
SIMPOSIO DISPLASIA BRONCO PULMONAR

Coordinadora: Dra. María Angélica Palomino

Salón 2

INTRODUCCIÓN

Dra. María Angélica Palomino

¿COMO DEFINIMOS DISPLASIA BRONCOPULMONAR?¿CUÁL ES LA MAGNITUD DEL PROBLEMA EN CHILE?

Dra. Mónica Morgues

¿QUÉ ES NORMALIDAD EN UNA OXIMETRÍA CONTINUA DE PULSO EN RECIÉN NACIDOS PREMATUROS?

Dra. Alejandra Zamorano

PATRONES DE OXIMETRÍA Y EVOLUCIÓN EN EL PRIMER AÑO DE VIDA Dra. Mónica Morgues

¿CUÁL ES EL IMPACTO DE LAS NUEVAS LECHES EN EL CRECIMIENTO SOMÁTICO Y PULMONAR DEL RECIÉN NACIDO?

Dra. Patricia Vernal

¿SE JUSTIFICA EL USO PROGRAMÁTICO DE PROFILAXIS DE VRS EN CHILE?

Dra. María Angélica Palomino

¿QUÉ ESPERAMOS DE LA FUNCIÓN PULMONAR Y CALIDAD DE VIDA EN EL LARGO PLAZO?

Dr. Héctor Gutiérrez

PREGUNTAS Y CONCLUSIONES

SIMPOSIO: PARED TORÁCICA

Coordinador: Dr. Rafael Prats

Salón A

ANATOMÍA QUIRÚRGICA DE LA PARED TORÁCICA

Dr. Raúl Berríos

MALFORMACIONES MÁS FRECUENTES DE PARED TORÁCICA

Dr. Patricio Varela

CIRUGÍA DEL PECTUS EXCAVATUM. ALTERNATIVAS TÉCNICAS

Dr. Marlos De Souza Coelho

CIRUGÍA DEL PECTUS EXCAVATUM. TÉCNICA CLÁSICA

Dr. Rafael Prats

CIRUGÍA DEL PECTUS EXCAVATUM. TÉCNICA DE NUSS

Dr. Patricio Varela

CIRUGÍA DEL PECTUS CARINATUM

Dr. Marlos de Souza Coelho

TUMORES DE LA PARED TORÁCICA

Dr. José Miguel Clavero

DISCUSIÓN

COCKTAIL

21:00 CENA - PRESENTACIÓN DE INVITADOS

Dra. Carolina Herrera y Dra. Isabel Valdés 


\section{JUEVES 6 DE NOVIEMBRE}

Coordinadores del día:

Coordinador Adulto: Dra. Patricia Schönffeldt

Coordinador Pediatra: Dra. Ana María Herrera

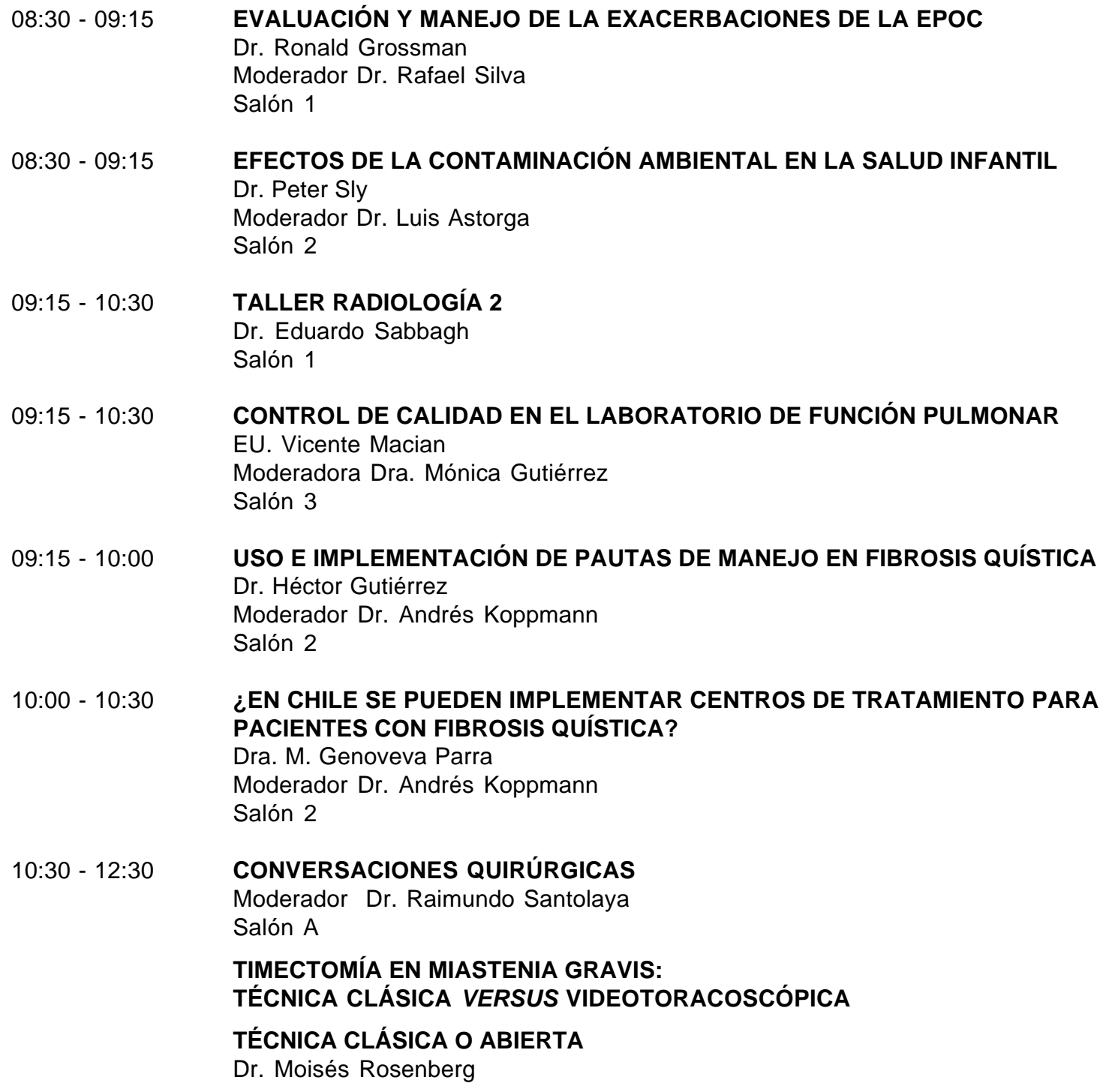

MESA REDONDA: SIMPATECTOMÍA

Café 
11:00 - 12:15 COMUNICACIONES LIBRES 1

$\begin{array}{lll}\text { SALÓN } & \text { № } 1 \text { (CL1 a CL5 ) } \\ \text { SESIÓN 1 } & \text { : } & \text { FUNCIÓN PULMONAR Y DAÑO PULMONAR EN PEDIATRIA } \\ \text { Coordinadores: } & \text { Drs. Andrés Koppmann y Viviana Aguirre }\end{array}$

CL-1

OXIMETRÍA CONTINUA DE PULSO (OCP) EN PREMATUROS < 32 SEMANAS ANTES DEL ALTA. ¿QUÉ ES LO NORMAL?. Morgues M., Vernal P., Palomino MA., Valdés I. y Montes S. Hospital San José, Universidad de Chile.

CL-2

RELACIÓN ENTRE LA RESPUESTA VEF VE, AL BRONCODILATADOR Y LA PRUEBA DE PROVOCACIÓN BRONQUIAL CON METACOLINA EN PACIENTES DE 3 A 15 AÑOS. Alvarez C., Gómez P., Aguilera J., Delgado I. y Corrales R. Servicio Laboratorio Broncopulmonar Clínica Alemana. Facultad de Medicina. Clínica Alemana-Universidad del Desarrollo. Santiago. Chile.

CL-3

EXPERIENCIA EN TRAQUEOSTOMÍA EN UN CENTRO DE HOSPITALIZACIÓN PROLONGADA: CARACTERIZACIÓN CLÍNICA. Montes S., Barañao P., Jakubson L., Salinas P., Rosales A. y Prado F. Hospital Josefina Martínez, Santiago. Chile.

CL-4

QUISTE BRONCOGÉNICO COMO CAUSA DEL SÍNDROME DE DIFICULTAD RESPIRATORIA EN RECIÉN NACIDO DE TERMINO. Fielbaum O., Varela P. y Martínez J.L. Centro Respiratorio Pediátrico Clínica Las Condes.

CL-5

GENERADORES DE FLUJO A TRAVÉS DE TRAQUEOSTOMÍA PARA VENTILACIÓN PROLONGADA CRÓNICA. Montes S., Barañao P., Salinas P., Méndez M. y Prado F. Hospital Josefina Martínez, Santiago. Chile.

SALÓN : № 2 (CL-6 a CL-10)

SESIÓN 1 : ASISTENCIA VENTILATORIA EN PEDIATRIA

Coordinadores: Drs. Selim Abara y Oscar Fielbaum

CL-6

EFECTO INMEDIATO DE LA KINESITERAPIA RESPIRATORIA EN EL TRATAMIENTO AMBULATORIO DEL SÍNDROME BRONQUIAL OBSTRUCTIVO. Espinoza C., Ferretti C., Manríquez P. y Ciudad D. Carrera de Kinesiología, Facultad de Medicina Universidad de Valparaíso. Consultorio Barón. Corporación Municipal de Valparaíso.

CL-7

EVALUACIÓN DE CALIDAD DE VIDA EN NIÑOS Y FAMILIA Y/O CUIDADORES BENEFICIARIOS DEL PROGRAMA NACIONAL DE VENTILACIÓN NO INVASIVA EN ATENCIÓN PRIMARIA DE SALUD. Salinas P., Farías A., Rodríguez C., Rosales A., Díaz K., Prado F., Mancilla P. y Astudillo P. Programa de Asistencia Ventilatoria No Invasiva, Unidad de Salud Respiratoria-Ministerio de Salud.

CL-8 PROGRAMA NACIONAL DE ASISTENCIA VENTILATORIA NO INVASIVA DOMICILIARIA EN ATENCIÓN PRIMARIA DE SALUD: UN AÑO DE EXPERIENCIA. Salinas P., Farías A., Vera R., Prado F., Mancilla P. y Astudillo P. Programa de Asistencia Ventilatoria No Invasiva, Unidad de Salud Respiratoria-Ministerio de Salud.

CL-9

RESOLUCIÓN DE EVENTOS DE LOS USUARIOS DEL PROGRAMA DE ASISTENCIA VENTILATORIA NO INVASIVA DEL MINISTERIO DE SALUD DE CHILE, PERÍODO 2006-2008. Farías A., Prado F., Salinas P., Mancilla P. y Astudillo P. Programa de Asistencia Ventilatoria no Invasiva. Unidad de Salud Respiratoria/ Subsecretarías de Redes.

CL-10

REDUCCIÓN DE HOSPITALIZACIONES EN LOS USUARIOS DEL PROGRAMA DE ASISTENCIA VENTILATORIA NO INVASIVA EN DOMICILIO DEL MINISTERIO DE SALUD DE CHILE, PERÍODO 2006-2008. Farías A., Salinas P., Prado F., Astudillo P. y Mancilla P. Centro Respiratorio Pediátrico, Clínica Las Condes. 
SALÓN : № 3 (CL-11 a CL-15)

SESIÓN 1 : FUNCION PULMONAR EN ADULTOS

Coordinadores: Drs. Henry Olivi y Patricia Schönffeldt

CL-11 DIFERENCIAS EN LA FUNCIÓN CARDIORRESPIRATORIA ENTRE PACIENTES ADULTOS MAYORES SECUELADOS DE ACCIDENTE CEREBROVASCULAR ISQUÉMICO Y ADULTOS MAYORES SIN LA PATOLOGÍA. Castro J., Fleming F. y Grossi M. Carrera de Kinesiología. Facultad de Medicina. Universidad de Valparaíso. Hospital de Quilpué. Servicio de Salud Viña del Mar-Quillota.

CL-12

CL-13

CL-14

CL-15

CL-16

CL-17

CL-18

CL-19

FUNCIÓN PULMONAR EN PACIENTES OBESOS CON Y SIN HIPOVENTILACIÓN DIURNA. Maquilón C., Antolini M., Contreras J.E., Moreno A. y Lira P. Instituto Nacional del Tórax y Hospital del Salvador.

CALIDAD DE ESPIROMETRÍAS REALIZADAS EN LA ATENCIÓN PRIMARIA DE SALUD. Cartagena C., Martínez F. y Barros M. Unidad de Enfermedades Respiratorias, Servicio de Medicina Interna, Hospital Van Buren y Escuela de Medicina, Universidad de Valparaíso.

ECUACIONES DE REFERENCIA ESPIROMÉTRICA EN POBLACIÓN ADULTA CHILENA SER 2008. Gutiérrez M., Valdivia G., Villarroel L., Contreras G., Cartagena C. y Lisboa C. Hospital Naval Viña del Mar, Departamentos Salud Pública y Enfermedades Respiratorias Pontificia Universidad Católica de Chile, Asociación Chilena de Seguridad y Hospital Van Buren, Valparaíso.

VALIDACIÓN VALORES DE REFERENCIA ESPIROMÉTRICA, SER 2008. Gutiérrez M., Valdivia G., Villarroel L., Contreras G., Cartagena C. y Lisboa C. Lab. Función Pulmonar Hospital Naval Viña del Mar, Departamentos Salud Pública y Enfermedades Respiratorias Pontificia Universidad Católica de Chile, Asociación Chilena de Seguridad y Hospital Van Buren, Valparaíso.

SALÓN : A (CL-16 a CL-20)

SESIÓN 1 : INVESTIGACIÓN BÁSICA

Coordinadores: Drs. María Angélica Palomino y Ricardo Pinto

DAÑO PULMONAR INDUCIDO POR LA EXPOSICIÓN AGUDA A SULFATO DE COBRE Y OZONO: EFECTO DE LA SECUENCIA DE EXPOSICIÓN. Sánchez S.*, Dussaubat N., Miller M.E., Medina C., González S. y Oyarzún M. Pg. Fisiopatología, ICBM, Facultad de Medicina, Universidad de Chile y Dept. Anatomía Patológica, Facultad de Medicina, Pontificia Universidad Católica de Chile.

COMPARACIÓN DE LA PRODUCCIÓN DE INTERFERON FRENTE A DIFERENTES ANTÍGENOS DE MYCOBACTERIUM, ENTRE NIÑOS CONTACTOS DE TUBERCULOSIS Y NIÑOS VACUNADOS CON BCG. Casar C., Hernández M., Castro-Rodríguez J.A., Mamani N., Pizarro P., Rueda B., Gallardo A. y Pierry C. Hospital Roberto del Río, Departamento Microbiología, Facultad Medicina Universidad de Chile, Hospital El Pino, Hospital San Juan de Dios.

PÉRDIDA DE GLUTATION DESDE PULMONES DE CONEJO EN SOLUCIÓN DE PRESERVACIÓN PARA TRASPLANTE. Solovera M.E., Cheyre J.E., Vecchiola A. y Borzone G. Sección de Cirugía de Tórax, Departamento de Enfermedades Respiratorias y Centro de Investigaciones Médicas, Pontificia Universidad Católica de Chile.

MAGNITUD DEL ENFISEMA INDUCIDO POR ELASTASA: POSIBLE RELACIÓN CON EL TIPO DE DAÑO AGUDO PULMONAR: Vecchiola A., Ramírez A., Villagrán A., Salazar I., León A. y Borzone G. Departamento de Enfermedades Respiratorias y Centro de Investigaciones Médicas, Pontificia Universidad Católica de Chile. 
CL-20

CL-21

CL-22

CL-23

CL-24

CL-25

$12: 15-13: 00$

$12: 15-13: 00$

$13: 00-14: 30$

$13: 00-14: 30$
POLIMORFISMOS EN GENES DE INTERLEUQUINAS (IL) 4 Y 8 DETERMINAN ENFEMEDAD GRAVE POR VIRUS RESPIRATORIO SINCICIAL Y SU EVOLUCIÓN A LARGo PLAZO. Tapia L, Ampuero S., Palomino MA., Ayarza E., Aguilar N. y Larrañaga C. Programa de Virología, ICBM y Departamento de Pediatría Norte, Universidad de Chile. Unidad de Broncopulmonar, Hospital Roberto del Río.

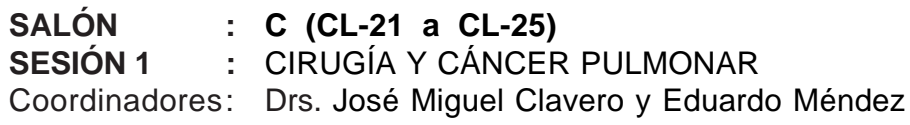

CAUSA POCO FRECUENTE DE DOLOR TORAXICO: HEMATOMA ESPONTÁNEO DE PARED. Czischke K., Marcone P., Oyonarte M., Céspedes I. y Norero B. Instituto Nacional del Tórax.

DISNEA POSTNEUMONECTOMÍA. Linacre V., Rodríguez P., Florenzano M. y Sepúlveda C. Servicio Médico Quirúrgico Respiratorio, Instituto Nacional del Tórax.

BRONQUIECTASIAS, HIPERPLASIA TIMICA Y SÍNDROME DEL LÓBULO MEDIO: PRESENTACIÓN POCO FRECUENTE DEL SÍNDROME DE SJÖGREN. Barcos P., Fuschini L., Linacre V., Miranda G. y Chernilo S. Instituto Nacional del Tórax.

ETAPIFICACIÓN ANATÓMICA VERSUS RADIOLÓGICA EN PACIENTES CON DIAGNÓSTICO DE CÁNCER PULMONAR SOMETIDOS A CIRUGÍA RESECTIVA EN EL INSTITUTO NACIONAL DEL TÓRAX: EXPERIENCIA DE UN AÑO. Miranda G., Barcos M., Linacre V., Zapata S., Sabbagh E. y Meneses M. Servicio de Radiología, Anatomía Patológica y Médico Quirúrgico, Instituto Nacional del Tórax.

CASO CLÍNICO: QUISTES HIDATÍDICOS PULMONARES, DIFICULTADES DIAGNÓSTICAS A PROPÓSITO DE DOS CASOS. García V.H. y García H. Servicio de Neumología, Hospital Clínico Viedma, Cochabamba, Bolivia.

\section{EFECTOS DE LA COCAÍNA Y CRACK EN EL PULMÓN Y EN EL SISTEMA} INMUNE

Dr. Donald Tashkin

Moderador Dra. Patricia Fernández

Salón 1

MALFORMACIONES CONGÉNITAS DE LA PARED TORÁCICA. ESTUDIO Y MANEJO QUIRÚRGICO

Dr. Patricio Varela

Moderadora: Dra. Virginia Linacre

Salón A

\section{SIMPOSIOS ALMUERZO}

¿CUÁL ES LA MEJOR ESTRATEGIA PARA COMENZAR EL TRATAMIENTO EN UN NIÑO ASMÁTICO? Y ¿CÓMO DEBO MONITORIZARLO?

Dr. José Antonio Castro y Dr. Ricardo Pinto

Gentileza GlaxoSmithKline

Salón 3

SIMPOSIOS ALMUERZO

PUESTA AL DÍA EN EXACERBACIONES DE EPOC

Dr. Fernando Descalzi

Gentileza Astra Zeneca

Salón 5 


\begin{tabular}{|c|c|}
\hline $13: 00-14: 30$ & $\begin{array}{l}\text { TALLER: ENFERMEDADES INTERSTICIALES } \\
\text { Dr. Kevin Flaherty } \\
\text { Coordinador Dr. Álvaro Undurraga } \\
\text { Salón } 6\end{array}$ \\
\hline $14: 30-18: 30$ & Tarde Libre \\
\hline $18: 30-19: 30$ & $\begin{array}{l}\text { Vinos y quesos } \\
\text { Gentileza Astra Zeneca } \\
\text { Bar }\end{array}$ \\
\hline $19: 30-20: 15$ & $\begin{array}{l}\text { Conferencia Cultural } \\
\text { DR. HECTOR ORREGO PUELMA } \\
\text { "La pequeña historia del cine chileno" } \\
\text { Sra. Alicia Scherson V. (cineasta) } \\
\text { Salón } 1 \\
\text { Gentileza: Laboratorios Saval }\end{array}$ \\
\hline $20: 00$ & $\begin{array}{l}\text { Cena } \\
\text { Restaurant }\end{array}$ \\
\hline $23: 00$ & $\begin{array}{l}\text { Fiesta Temática (sombreros) } \\
\text { Discoteque }\end{array}$ \\
\hline
\end{tabular}




\section{VIERNES 7 DE NOVIEMBRE}

Coordinadores del día:

Coordinador Adulto: Dra. Patricio Jiménez

Coordinador Pediatra: Dra. Claudia Astudillo

08:30 - 09:15

09:15 - 10:30

$09: 15-10: 30$

09:15 - 10:30

$08: 30-10: 30$

$10: 30-11: 00$

$11: 00-12: 15$

CL-26

CL-27

CL-28

CL-29

CL-30
EFECTOS DE LA MARIHUANA EN EL PULMÓN Y EN EL SISTEMA INMUNE

Dr. Donald Tashkin

Moderador: Dr. Jorge Montenegro

Salón 1

CONSENSO SER VENTILACIÓN MECÁNICA NO INVASIVA

Drs. Orlando Díaz, Francisco Arancibia y Gerardo Ferrero

Moderador: Dr. César Maquilón

Salón C

\author{
AUTOEDUCACIÓN Y MANEJO EN ASMA Y EPOC ¿QUÉ ES Y CÓMO \\ FUNCIONA? \\ Dr. Martyn Partridge \\ Moderadora: Dra. Liliana Vicherat \\ Salón 1
}

ACTUALIDADES EN TERAPIA DEL ASMA BRONQUIAL INFANTIL

Comisión Asma Pediátrica

Moderador: Dr. Carlos Ubilla

Salón 2

\section{SESIÓN DE CASOS CLÍNICOS QUIRÚRGICOS}

Moderador: Dr. Cristián González

Dr. David Lazo, Dr. Alfonso Oropesa, Dr. Rafael Prats

Salón A

Café

\section{COMUNICACIONES LIBRES 2}

\author{
SALÓN : № 1 (CL-26 a CL-30) \\ SESIÓN 1 : ASMA EN NIÑOS \\ Coordinadores: Drs. Evelyn Chala y Carolina Cruz
}

FACTORES DE RIESGO DE ASMA NO ATÓPICA EN ESCOLARES DEL NIVEL PRIMARIO DE ATENCIÓN DE LA COMUNA DE PUENTE ALTO. Quiroz E., CastroRodríguez J., Monsalve P., Leisewitz T. y Valdivia G. Departamentos Medicina Familiar y Salud Pública, Pontificia Universidad Católica de Chile.

EXACERBACIÓN DE ASMA BRONQUIAL EN LA UNIDAD DE PACIENTE CRÍTICO: CARACTERIZACIÓN CLÍNICA. Herrera A.M., Saavedra M., Escobar A.M., Medina M.E., Salgado E., Palavecino T. y Kutz A.M. Departamento de Pediatría Norte, Facultad de Medicina, Universidad de Chile, Hospital Roberto del Río.

UN AÑO DE SEGUIMIENTO A NIÑOS HOSPITALIZADOS POR ASMA. González R., Zúñiga J., Campos C., Pérez M., Pavón D. y Manríquez J. Hospital Exequiel González Cortés. Facultad de Medicina Campus Sur, Universidad de Chile.

PERFIL EPIDEMIOLÓGICO EN NIÑOS ASMÁTICOS CON ANTECEDENTES DE HOSPITALIZACIONES Y DE NIÑOS CON ASMA CONTROLADOS EN EL NIVEL PRIMARIO. Campos C., Zúñiga J., González R., Pérez M., Ramírez A. y Manríquez J. Hospital Exequiel González Cortés. Facultad de Medicina Campus Sur, Universidad de Chile.

CORRELACIÓN ENTRE PARÁMETROS CLÍNICOS DE CONTROL DE ASMA EN PEDIATRÍA. Vidal A., Ubilla C., Huerta D. y Medina M. Hospital Roberto del Río. 
SALÓN : № 2 (CL-31 a CL-35)

SESIÓN 1 : FUNCIÓN PULMONAR EN ADULTOS (2)

Coordinadores: Drs. Juan Céspedes y Sylvia Palacios

CL-31 SOMNOLENCIA DIURNA EXCESIVA EN CONDUCTORES DE VEHÍCULOS DE ALTO TONELAJE. Salinas M., Riveros A. y Contreras G. Fundación Científica y Tecnológica, ACHS.

CL-32

FUNCIÓN PULMONAR EN PACIENTES PORTADORES DE FIBROSIS PULMONAR AL MOMENTO DE DERIVACIÓN A TRASPLANTE. Zapata S., Granjean J., Alba A. y Parada M.T. Clínica las Condes e Instituto Nacional del Tórax.

CL-33

SENSIBILIDAD Y ESPECIFICIDAD DE LA ESPIROMETRIA PARA EL DIAGNÓSTICO DE ALTERACIÓN RESTRICTIVA UTILIZANDO LOS VALORES DE REFERENCIA SER 2008. Gutiérrez C.M., Valdivia G., Villarroel L., Contreras G., Cartagena C. y Lisboa C. Laboratorio Función Pulmonar Hospital Naval Viña del Mar, Deptos. Salud Pública y Enfermedades Respiratorias Pontificia Universidad Católica de Chile, Asociación Chilena de Seguridad y Hospital Van Buren, Valparaíso.

CL-34

TEST CARDIOPULMONAR DE EJERCICIO: COMUNICACIÓN PRELIMINAR DE VALORES DE REFERENCIA EN POBLACIÓN DE ADULTOS DE NUESTRO PAÍS. Caviedes I., Soto R. y Gómez P. Laboratorio Broncopulmonar, Clínica Alemana de Santiago, Facultad de Medicina Clínica Alemana, Universidad del Desarrollo.

CL-35

RENDIMIENTO DE LA RELACIÓN VEF $\mathrm{VEF}_{6}$ COMPARADO CON VEF $_{1} /$ CVF EN LA DETECCIÓN DE OBSTRUCCIÓN BRONQUIAL EN EL INSTITUTO NACIONAL DEL TÓRAX. Melo J., Czischke K., Céspedes J., Schonffeld P., Barcos P., Miranda G. y Salamanca M. Laboratorio función pulmonar, Instituto Nacional del Tórax.

SALÓN : № 3 (CL-36 a CL-40)

SESIÓN 1 : TABAQUISMO

Coordinadores: Drs. Sergio Bello y Rodrigo Gil

CL-36 COMPARACIÓN DE LA EFECTIVIDAD DE UNA CAMPAÑA VIRTUAL ANTITABACO CON ORIENTACIÓN MÉDICA DE ALTO IMPACTO VERSUS LA ADVERTENCIA DISPONIBLE A NIVEL NACIONAL PARA DISMINUIR LA PREVALENCIA DE TABAQUISMO EN LOS ESTUDIANTES DE LA UNIVERSIDAD DIEGO PORTALES DE SANTIAGO DE CHILE. Alzamora F., Revuelta F., Agloni M., Martínez N., Navarro A. y Roine I. Facultad de Ciencias de la Salud, Universidad Diego Portales.

CL-37

COMPORTAMIENTO DE LAS CONSULTAS RESPIRATORIAS EN UN SERVICIO DE URGENCIA ADULTO DE LA REGIÓN METROPOLITANA. Ríos F. y Castillo S. Servicio de Salud Metropolitano Central.

CL-38

PROGRAMA DOMICILIARIO ASISTENCIA VENTILATORIA NO INVASIVA EN ADULTOS. Maquilón C., Antolini M., Cabrera O., Lira P., Muñoz J., Canales K., Olave C., Zulic C., Muñoz C., Cabezas C., Ecchio R. y Peñaloza G. Unidad Salud Respiratoria. Ministerio de Salud.

CL-39 EXPERIENCIA CON VARENICLINE EN CESACIÓN TABÁQUICA. Tapia S., Araneda C. y Tapia M. Hospital Las Higueras Talcahuano.

CL-40

IMPACTO DE UNA INTERVENCIÓN EN LA CESACIÓN DEL HÁBITO TABÁQUICO EN LOS COLEGIOS PARTICIPANTES DEL CONCURSO "EL QUE NO FUMA GANA" DE LA PROVINCIA DE QUILLOTA. Pavié J., Andrade A., Razeto M., Estay A. y Valle X. Hospital San Martín de Quillota; Centro Respiratorio Integral Quillota; Sala ERA Olmué y Melón. 


$\begin{array}{ll}\text { SALÓN } & \text { : A (CL-41 a CL-45) } \\ \text { SESIÓN 1 : PROBLEMAS CLÍNICOS (1) } \\ \text { Coordinadores: Drs. Alvaro Undurraga y Patricio Jiménez } \\ \text { CASO CLÍNICO. LINFANGIOLEIOMIOMATOSIS PULMONAR. Silva R., Puelma F., } \\ \text { Cruzat C., Retamal V. y Reyes C. Servicio de Medicina, Cirugía y Unidad de Respi- } \\ \text { ratorio Hospital Regional de Talca, Anatomía Patológica Escuela Medicina Univer- } \\ \text { sidad Católica del Maule y Servicio de Radiología Hospital Base Linares. }\end{array}$

CL-42 ACTINOMICOSIS Y CÁNCER PULMONAR EN UN PACIENTE INMUNODEPRIMIDO: REPORTE DE UN CASO. Jalilie A., Núñez G., Peña P., De Diego P. y Meneses M. Unidad de Pacientes Críticos, Unidad de Enfermedades Respiratorias, Departamentos de Radiología y Anatomía Patológica. Clínica Santa María.

CL-43 FALLA RESPIRATORIA POR ÁCIDO TRANS-RETINOICO. Chahuán M. e Intriago M. Servicio de Medicina, Hospital Clínico San Borja Arriarán. Departamento Medicina Centro, Facultad de Medicina, Universidad de Chile.

CL-44 LINFANGIOLEIOMIOMATOSIS UNA CAUSA INHABITUAL DE NEUMOTÓRAX ESPONTÁNEO. Linacre V., Sepúlveda C., Florenzano M., Fica M. y Meneses M. Servicio Médico Quirúrgico Respiratorio, Instituto Nacional del Tórax.

CL-45

HISTIOCITOSIS PULMONAR DE CÉLULAS DE LANGERHANS: SERIE DE CASOS. Miranda G., Barcos P. y Rodríguez J.C. Servicio de Medicina, Instituto Nacional del Tórax.

$\begin{array}{lll}\text { SALÓN } & \text { C (CL-46 a CL-50) } \\ \text { SESIÓN } 1 & : \text { FIBROSIS QUÍSTICA } \\ \text { Coordinadores: } & \text { Drs. María Angélica Pérez y María Genoveva Parra }\end{array}$

CL-46 FIBROSIS QUÍSTICA: ÁREA NORTE DE SANTIAGO-2008. Montes S., Parra G. y Lozano J. Hospital Roberto del Río, Santiago, Chile.

APLICACIÓN DE TÉCNICAS DE GESTIÓN DE CALIDAD MEJORA PARÁMETROS CLÍNICOS PREDICTIVOS DE SOBREVIDA EN PACIENTES CON FIBROSIS QUíSTICA. Britton L., Thrasher S., Mims C. y Gutiérrez H. Children's Health System; Division of Pulmonary Medicine, Department of Pediatrics, University of Alabama at Birmingham. Alabama. USA.

CL-48 TRASPLANTE PULMONAR EN FIBROSIS QUÍSTICA: EXPERIENCIA CHILENA. Fernández P. y Parada M T. Clínica Las Condes. Instituto Nacional del Tórax.

CL-49 FUNCIÓN PULMONAR EN NIÑOS CON FIBROSIS QUÍSTICA. Montes S., Escobar A.M., Valenzuela J.L., Parra G. y Lozano J. Hospital Roberto del Río, Santiago, Chile.

CL-50 EVALUACIÓN DEL PRIMER PROGRAMA DE TRANSICIÓN: FIBROSIS QUÍSTICA COMO MODELO. Fernández P., Navarro J., Jiménez P., Lozano J., Herrera O. y Astorga L. Instituto Nacional del Tórax, Hospital Luis Calvo Mackenna, Hospital Roberto del Río.

$12: 15-13: 00$

GUÍAS DE MANEJO DE NEUMONÍA

Dr. Ronald Grossman

Moderador: Dr. Mauricio Riquelme

Salón 1

$12: 15-13: 00$

MANUAL DE PROCEDIMIENTOS SER: PRUEBA DE CAMINATA DE 6 MINUTOS

Comisión de Función Pulmonar

EU. Vicente Macián

Moderadora: Dra. María Teresa Beroíza

Salón C 


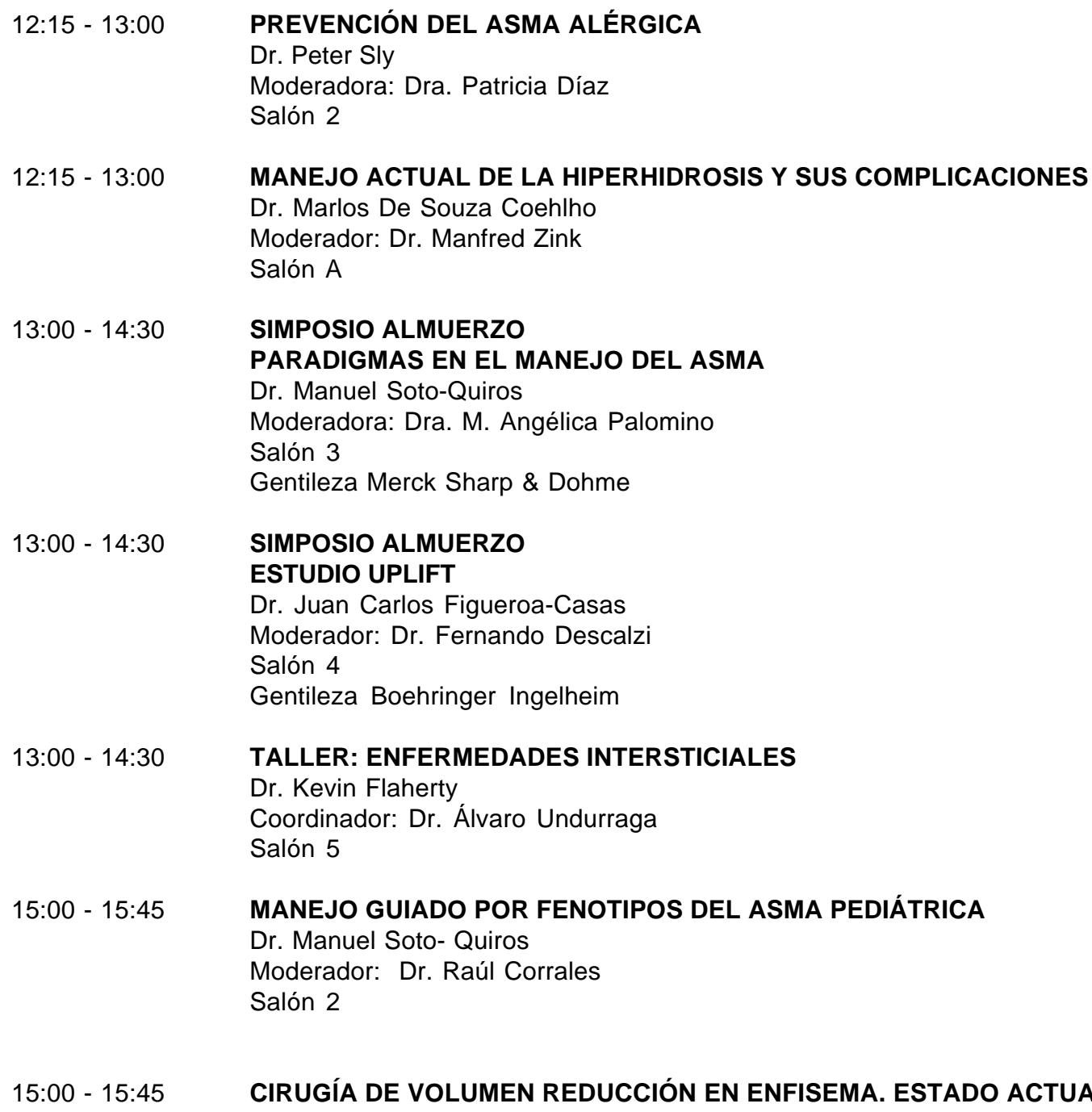

COMUNICACIONES LIBRES 3

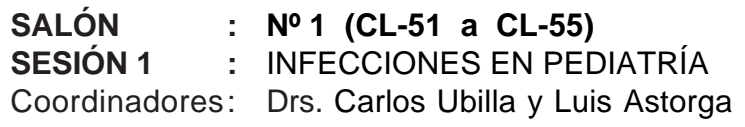

CL-51 METAPNEUMOVIRUS HUMANO, NUEVO AGENTE DE INFECCIÓN RESPIRATORIA BAJA EN LACTANTES HOSPITALIZADOS EN VALPARAÍSO. Henríquez M.T., Milinarsky A., Lezana V., Llanos J. y Vergara R. Hospital Carlos Van Buren y Universidad de Valparaíso.

CL-52 INFECCIÓN POR METAPNEUMOVIRUS HUMANO EN NIÑOS HOSPITALIZADOS POR UNA ENFERMEDAD RESPIRATORIA AGUDA BAJA GRAVE. Lozano J., Yáñez L., Lapadula M., Lafourcade M., Herrada L., Budnik I. y Burgos F. Unidad de Paciente Crítico Pediátrico Clínica Santa María.

CL-53 CASO CLÍNICO PLEURONEUMONÍA COMPLICADA. Mora E. y Sanhueza A. Hospital de Carabineros. 

QUIAL OBSTRUCTIVO RECURRENTE O ASMA BRONQUIAL: EXPERIENCIA CLÍNICA EN UNA COHORTE CHILENA. Tapia L., Mamani R., Larrañaga C., Ampuero S. y Palomino M.A. Departamento de Pediatría Norte, Universidad de Chile. Unidad de Broncopulmonar, Hospital Roberto del Río.

CL-55

DETECCIÓN DE PNEUMOCYSTIS JIROVECI EN NIÑOS INMUNOCOMPETENTES MENORES DE 6 MESES CON BRONQUIOLITIS Y/O NEUMONIA ADQUIRIDA EN LA COMUNIDAD. Palomino M.A., Ponce C.A., Ubilla C., Pizarro P., Saavedra M., Herrera A.M., Ruiz A., Mackenney J., Araya A., Bustamante R., Vargas S.L. Departamento de Pediatría, Facultad de Medicina Campus Norte, Universidad de Chile. Laboratorio de Infecciones Respiratorias, Programa de Microbiología ICBM, Facultad de Medicina Universidad de Chile.

\author{
SALÓN : № 2 (CL-56 a CL-60) \\ SESIÓN 1 : EPOC Y ASMA ADULTO \\ Coordinadores: Drs. Edgardo Cruz y Rosa María Feijoo
}

CL-56

EXACERBACIONES EN PACIENTES EX-FUMADORES CON ENFERMEDAD PULMONAR OBSTRUCTIVA CRÓNICA: EFECTOS FUNCIONALES Y CLÍNICOS A CORTO PLAZO. Sandoval C., Dreyse J., Díaz O., Saldías F. y Lisboa C. Departamento de Enfermedades Respiratorias. Pontificia Universidad Católica de Chile.

CL-57 MARCADORES DE INFLAMACIÓN SISTÉMICA EN LA ENFERMEDAD PULMONAR OBSTRUCTIVA CRÓNICA ESTABLE. Lisboa C., Morales A., Dreyse J., Saldías F. y Díaz O. Departamento de Enfermedades Respiratorias. Pontificia Universidad Católica de Chile.

CL-58

ASOCIACIÓN ENTRE CONTROL, GRAVEDAD Y CALIDAD DE VIDA EN PACIENTES ASMATICOS. Barros M., González R., González N. y Anderson J. Escuela de Medicina, Universidad de Valparaíso y Servicio de Medicina Interna, Hospital Van Buren, Valparaíso.

CL-59

COMORBILIDAD EN PACIENTES CON ENFERMEDAD PULMONAR OBSTRUCTIVA CRÓNICA SEVERA. Contreras J., Solís E., Rubilar S., Matus O. y Palacios S. Sala ERA, Sección Broncopulmonar, Hospital G Grant B, Gestión Atención Primaria, Servicio Salud Concepción. Departamento Educación Médica, Facultad Medicina, Universidad de Concepción.

CL-60

TEST CARDIOPULMONAR: DETERMINACIÓN DE LIMITANTES AL EJERCICIO A NIVEL DE UMBRAL VENTILATORIO EN PACIENTES CON EPOC. Caviedes I., Soto R. y Gómez P. Laboratorio Broncopulmonar, Clínica Alemana de Santiago, Facultad de Medicina Clínica Alemana, Universidad del Desarrollo.

$\begin{array}{ll}\text { SALÓN } & \text { № } 3 \text { (CL-61 a CL-65) } \\ \text { SESIÓN 1 } & : \text { MISCELÁNEO } \\ \text { Coordinador } & : \text { Dr. Juan Carlos Rodríguez }\end{array}$

CL-61 TRASPLANTE PULMONAR EN PACIENTES PORTADORES DE FIBROSIS PULMONAR IDIOPATICA: SEGUIMIENTO A LARGO PLAZO. Parada M.T., Zapata S., Granjean J. y Alba A. Clínica Las Condes e Instituto Nacional del Tórax.

CL-62

NEUMONÍA ADQUIRIDA EN LA COMUNIDAD GRAVE, 6 AÑOS DE EXPERIENCIA EN LA UCI DEL INSTITUTO NACIONAL DEL TÓRAX. Arancibia F., Grandjean J., Rivas F., Salamanca L., Graver L. y Soto L. UCl y Laboratorio Microbiología, Instituto Nacional del Tórax. 

TULA BRONCOPLEURAL. Marcone P., Linacre V. y Sepúlveda C. Instituto Nacional del Tórax. DE ESPERA DE TRASPLANTE PULMONAR. Ibarra C. y Parada M.T. Centro de Trasplantes, Clínica Las Condes. DESCAMATIVA. García V.H. y Burgos R. Servicio de Neumología y Cirugía de Tórax, Hospital Clínico "Viedma". Cochabamba, Bolivia.

\author{
SALÓN : A (CL-66 a CL-70) \\ SESIÓN 1 : PROBLEMAS CLÍNICOS (2) \\ Coordinadores: Drs. Maite Oyonarte y Mónica Zagolín
}

CL-66 HIPERTENSIÓN PULMONAR EN HEMODIÁLISIS CRÓNICA. Rioseco P., Rubilar M., Verbal F., Pedreros C., Morales V., López R. y Salazar P. Medicina Interna Hospital Las Higueras Talcahuano, Chile.

CL-67

DETERMINACIÓN DE COLESTEROL EN LÍQUIDO PLEURAL COMO HERRAMIENTA DIAGNÓSTICA EN DERRAMES PLEURALES. Valenzuela H., Monsalve L., Oyanadel M.L., Martínez F. y Carrizo A. Universidad de Valparaiso, Hospital Dr. Eduardo Pereira.

CL-68

VASORREACTIVIDAD A ADENOSINA EN LOS PACIENTES CON HIPERTENSIÓN PULMONAR ARTERIAL: PREVALENCIA Y RESPUESTA CLÍNICA, FUNCIONAL Y HEMODINÁMICA AL TRATAMIENTO CON DILTIAZEM. Zagolín M., Czischke K., Uriarte P., Medel N., Parra C. y Vargas D. Instituto Nacional del Tórax.

SíNDROME EOSINOFÍLICO PULMONAR. CASO CLÍNICO. Miranda G., Barcos P., Zapata S. y Arancibia F. Servicio de Medicina, Instituto Nacional del Tórax.

CL-70

SOLICITUD DE ANGIOTAC POR SOSPECHA DE TEP EN TRABAJO RUTINARIO DEL SERVICIO DE MEDICINA DEL INT. Barcos P., Zapata S., Miranda G. y Sabbagh E. Servicio de Radiología y Servicio Médico Quirúrgico, Instituto Nacional del Tórax.

\author{
SALÓN : C (CL-71 a CL-75) \\ SESIÓN 1 : TUBERCULOSIS \\ Coordinadores: Drs. Victorino Farga y Carlos Casar
}

CL-71 NOCARDIOSIS PULMONAR Y TUBERCULOSIS EN PACIENTE PORTADOR DEL VIH. CASO CLÍNICO. Leyton J., Monsalve L., Oyanadel M.L. y Cruz R. Hospital Eduardo Pereira. Escuela de Medicina, Universidad de Valparaíso.

CL-72 ESPECTRO CAMBIANTE DE LA TBC EN CHILE. Muñoz S., Rioseco P. y Rubilar M. Enfermedades Respiratorias, Hospital Las Higueras, Talcahuano, Chile.

CL-73

PACIENTE INMUNOCOMPETENTE CON TBC PULMONAR BK (+) VT: POSTERIOR A DOS TRATAMIENTOS EFICIENTES CONTINÚA BK (+) Y CULTIVO DE KOCH (+). Mendoza K., Saldías F., Meneses M. y Jiménez P. Sub Departamento de Medicina, Hospital Clínico Regional Valdivia; Instituto de Medicina, Universidad Austral de Chile. 
tal G Grant B, Concepción. Departamento Educación Médica, Facultad Medicina, Universidad de Concepción.

CL-75 TUBERCULOSIS RECTAL QUE SIMULA CÁNCER RECTAL. García V.H., Vargas G. y Villalpando M. Servicio de Neumología y Gastroenterología, Hospital Clínico "Viedma", Cochabamba, Bolivia.

17:00-17:30 Café

17:30 - 19:30 Controversias Dr. Patricio González

Moderador: Dr. Juan Carlos Rodríguez

Salón 1

1. ¿SE JUSTIFICA LA BIOPSIA PULMONAR EN LA ENFERMEDAD INTERSTICIAL ASOCIADA A MESENQUIMOPATÍA?

Pro : Dr. Matías Florenzano

Contra : Dr. Carlos Inzunza

2. ¿TIENE HOY ALGÚN ROL LA INMUNOTERAPIA EN EL ASMA BRONQUIAL? Pro : Dr. Fanor Villanueva

Contra : Dra. Rosa María Feijoo

3. ¿UN PET(+) EN N2 HACE INNECESARIA LA MEDIASTINOSCOPÍA?

Pro : Dr. David Ladrón De Guevara

Contra : Dr. Felipe Bannura

4. ¿EL TRATAMIENTO MÉDICO PRECOZ DE LA PLEURONEUMONÍA EN EL NIÑO CON FIBRINOLÍTICOS ES DE ELECCIÓN AL TRATAMIENTO QUIRÚRGICO?

Pro : Dr. José Perillan

Contra : Dr. Rodrigo Bozzo

19:30 - 20:30

SIMPOSIO

LA FRONTERA ASMA-EPOC: ¿UNA ENTIDAD DIFERENTE?

Dr. Ricardo Sepúlveda

Salón A

Gentileza GlaxoSmithKline

19:30 - 20:30 SIMPOSIO

REALIDAD ACTUAL Y APLICACIONES PRÁCTICAS EN EL MANEJO DEL ASMA

Dr. Martyn Partridge

Coordinadora: Dra. Patricia Díaz

Salón C

Gentileza: Astra Zeneca

20:30-21:15 Vinos y quesos

Gentileza Astra Zeneca

Bar

21:15

Cena de Clausura

(Tenida Formal)

Salón 1 


\section{SÁBADO 8 DE NOVIEMBRE}

Coordinadores del día:

Coordinador Adulto: Dr. Pablo Marcone

Coordinador Pediatra: Dr. Andrés Koppmann

09:00 - 09:45

LIMITACIÓN DEL ESFUERZO TERAPÉUTICO EN PACIENTE RESPIRATORIO TERMINAL

Dra. Carolina Herrera

Moderador: Dr. Edgardo Cruz

Salón 1

$09: 45-10: 45$

TALLER: VENTILACIÓN MECÁNICA NO INVASIVA

Moderador: Dr. César Maquilón

1. TOS ASISTIDA

Dr. Gerardo Ferrero

2. VENTILACIÓN CONTRA RESISTENCIA

Dr. Guillermo Montiel

Salón 3

09:45 - 10:45 OPCIONES TERAPÉUTICAS EN FIBROSIS PULMONAR: HOY Y MAÑANA

Dr. Kevin Flaherty

Moderador: Dr. Álvaro Undurraga

Salón 1

$09: 45-10: 45$

TRANSICIÓN DE ADOLESCENTE A ADULTO EN FIBROSIS QUÍSTICA

Dr. Héctor Gutiérrez

Moderadora: Dra. Alexis Strickler

Salón 2

10:45 - 11:15 Café

11:15 - 12:30 TALLER: VENTILACIÓN MECÁNICA NO INVASIVA

Moderador: Dr. César Maquilón

1. VENTILADOR VISIÓN

Dr. Felipe Aller

2. VENTILACIÓN POR TRAQUEOSTOMÍA

EU. Pamela Salinas

Salón 3

$11: 15-12: 30$

TALLER: ENFERMEDADES INTERSTICIALES: CASOS CLÍNICOS

Coordinador: Dr. Álvaro Undurraga

Salón 1

11:15 - 12:30 CASOS CLÍNICOS DE FIBROSIS QUÍSTICA

Coordinadora: Dra. María Angélica Pérez

Salón 2

11:15 - 12:30 TUMORES NEUROENDOCRINOS DEL PULMÓN

Dr. Moisés Rosenberg

Moderador: Dr. Alfonso Oropesa

Salón A

$12: 30-13: 00$

REUNIÓN INFORMATIVA DE SOCIOS

Dra. Patricia Schonffeldt

Salón 1

13:00 - 13:15 Clausura

Preside: Dr. Fernando Rivas

Salón 1

14:00 Asado campestre

Gentileza Astra Zeneca - Restaurant 